\title{
INCIDENCIA DE LOS PECES PARA CONSUMO HUMANO DIRECTO EN LAS PESQUERÍAS INDUSTRIALES DEL LITORAL SURPERUANO
}

\author{
Luis A. Flores Portugal ${ }^{1}$, Jorge Fernández R. ${ }^{2}$
}

\author{
R E S U M E N
}

Los pescadores artesanales siempre han presentado quejas a las autoridades del sector pesquero, dejando sentir sus protestas por la competencia desleal de la flota industrial; que en ciertos momentos opera muy cerca de la costa, capturando buenos volumenes de peces que deben ser destinados para el consumo humano directo.

Los objetivos del trabajo son: a) Establecer en qué medida la flota industrial opera en áreas donde pescan regularmente para consumo y artesanal. b) Cual es la incidencia de los peces para consumo, dentro de las pesquerias industriales en la zona sur del litoral periano.

El periodo del estudio fue desde noviembre 1994 hasta octubre 1995 y las fuentes de información fueron: a) Observando, a bordo de las bolicheras, la composicion de las capturas en las áreas de pesca. b) Los partes diarios de desembarques de cada embarcación, que Pesca Perí remite al IMARPE-Ilo, donde indica: arreas de pesca, volumenes capturados y composicion de las capturas.

Durante el uño del estudio se desembarcaron en la zona sur 620,164.3 tm y la incidencia de peces para consumo, principalmente jurel juvenil, fue de $2.65 \%$.

Las áreas de mayor producción fueron consideradas afuera de las 3 millas de la cosia y la competencia desleal prácticamente no existió nunca.

\section{A B S T R A C T}

The objectives of this work are: a) to establish, until what extent, industrial fleet work in areas where fish are regularly caught for non-industrial consumption and b) which is the incidence of fish for consumption within industrial fisheries in the south zone of the peruvian coast.

The time for the study, was from november 1994 to october 1995 and the information resources were: a) watching from small boats, daily report of unloading from every fishing areas and b) the daily report of unloading from every fishing boat, which Pesca Perú, sends to IMARPE-llo, indicating: fishing areas, volumen captured and composition of the capture.

During a year of study, 620, $164.3 \mathrm{tmb}$ were unloaded in the south zone. and the incidence of fish for consumption, mainly, young mackarel was $2.65 \%$. The major production areas were considered to be 3 miles away from the coast and the unfair competence never existed.

\section{INTRODUCCIÓN}

El ministerio de pesquería, en atención de múltiples solicitudes y reclamos de la Federación de Pescadores Artesanales a lo largo del litoral peruano,
1. Biólogo
2. Técnico pesquero

en el año de 1991, emitió la Resolución Ministerial Nro. 172-92-PE; que en su parte resolutiva disponía que la flota industrial no debería operar dentro de las cinco primeras millas y asi evitar una competencia desleal con las flotas para consumo y artesanal. Esta disposición que, en su concepción, generalizaba el modo operativo de la flota industrial, sin tener en consideración que el comportamiento de los recursos 
pesqueros, especialmente aquéllos considerados como pelágicos, entre los cuales se encuentra el recurso 'anchoveta' (Engraulis ringer J.), que es la materia prima para la transformación de harina y aceite de pescado.

La conformación, concentración y distribución de los cardúmenes y por lo tanto su comportamiento, no es igual a lo largo de la franja costera del litoral peruano; pues mientras en la zona norte desde Paita hasta Huarmey $\left(05^{\circ}\right.$ a $10^{\circ}$ L.S.); a la anchoveta se le captura hasta mas allá de 80 millas afuera de la costa, en la zona sur, desde San Juan hasta la frontera sur $\left(15^{\circ}\right.$ a $18^{\circ} 20^{\prime}$ L.S.), más del $80 \%$ de las capturas se ejecutan dentro de las 10 millas afuera de la costa.

Esta situación ha sido el principal objetivo que llevó a los autores a realizar el presente estudio y así establecer, cuál es la incidencia de peces, considerados para consumo humano, dentro de las pesquerías industriales y cuál es la interferencia entre la flota industrial con las flotas para consumo y artesanal.

\section{MATERIALES Y MÉTODOS}

El periodo del estudio ha sido desde noviembre de 1994 hasta octubre de 1995, en la zona comprendida desde Camaná (16 $45^{\circ}$ L.S.), hasta la frontera sur $\left(18^{\circ} 20^{\prime}\right.$ L.S.). Tres han sido las fuentes de información:

a) Salidas al mar a bordo de las embarcaciones de la flota industrial, realizando observaciones, sobre las áreas de pesca, distancia hacia la costa donde se ejecutan las capturas, profundidad del lugar de pesca y la composición por especies de las capturas realizadas, mediante muestreos.

b) Los partes diarios de operación de la flota industrial, que la oficina de bahía de la empresa Pesca Perú, remite a la dirección del laboratorio costero del IMARPE - Ilo. En dichos partes se informa sobre el número de embarcaciones que salieron en la fecha a pescar, las áreas donde pescaron, los volúmenes desembarcados por cada embarcación y la composición por especies de la capturas realizadas.

c) Los muestreos diarios que personal del IMARPE Ilo, realiza en las plantas transformadoras de la empresa Pesca Perú; allí toman muestras del 30\% de la flota que desembarca cada día, observando la composición por especies de las capturas y obtienen información sobre áreas de pesca, distancia a la costa, etc.

\section{III.RESULTADOS}

\subsection{DEL MEDIO AMBIENTE}

Durante la estación de verano, diciembre febrero, se desarrolló sumamente oscilante, en relación con las temperaturas y los vientos, factores que hicieron que el comportamiento de los recursos pesqueros, principalmente los considerados peces pelágicos, como la anchoveta, que realiza migraciones tróficas en busca de alimento y de condiciones ambientales que le sean favorables para su desarrollo vital.

Los registros de temperatura en la superficie del mar oscilaron con un mínimo de $14.8^{\circ} \mathrm{C}$ en noviembre - 1994 y un máximo de $22.6^{\circ} \mathrm{C}$ en enero - 95 y con promedios mensuales de $16.3^{\circ}$ en noviembre; $17.3^{\circ}$ en Diciembre y $18.2^{\circ} \mathrm{C}$ en enero95 respectivamente.

- En el periodo enero a marzo, sucedieron oscilaciones algo notables en los registros de temperaturas de hasta $4^{\circ} \mathrm{C}$ en periodos muy cortos de 24 horas, pero los afloramientos de aguas frías, procedentes de la corriente subantártica, lo cual en cierta medida permitió que la presencia y acción de las aguas oceánicas con características de subtropicales superficiales (STS), no sean tan impactantes sobre los recursos pesqueros, principalmente sobre la 'anchoveta'.

En el área de Ilo, las temperaturas en la superficie del mar oscilaron desde un mínimo de $15.8^{\circ} \mathrm{C}$ hasta un máximo de $20.1^{\circ} \mathrm{C}$, con promedios mensuales de $17.8^{\circ} \mathrm{C}$ en febrero y de $17.5^{\circ} \mathrm{C}$ en marzo.

Durante el otoño, de abril a junio, el medio ambiente marino fue muy cambiante, posiblemente por la presencia mas notable de la corriente subantártica, lo que motivó que la 'anchoveta' tuviera un comportamiento de gran dispersión de los cardúmenes y la entrada en las pesquerías del recurso potencial llamado 'camotillo' (Normanichthys crockeri G.) sobre todo en el mes de abril.

Los registros de temperatura oscilaron con un mínimo de $15.0^{\circ} \mathrm{C}$ en junio y con un máximo de $18.1^{\circ} \mathrm{C}$ en abril con promedio mensuales de $16.8^{\circ}$ en abril y 15.7 en mayo y junio.

- En el periodo de julio a setiembre, el ambiente marino en el área de 1 lo, los registros de temperaturas en la superficie del mar presentaron valores que oscilaron con un mínimo de $13.8^{\circ}$ en setiembre y $16.1^{\circ} \mathrm{C}$ en julio, con promedios de 
$15.4^{\circ} \mathrm{C}$ en julio; $14.5^{\circ} \mathrm{C}$ en agosto y $14.7^{\circ} \mathrm{C}$ en setiembre. Este ambiente frío en el mar permitió la ausencia casi total de las pesquerías del recurso anchoveta y la sustitución por el recurso 'camotillo' y la baja sensible en los volúmenes capturados en la zona sur del litoral peruano.

\subsection{DE LOS RECURSOS PESQUEROS}

Se ejecutó un análisis de la información obtenida en forma mensual sobre las áreas de pesca, volúmenes capturados y composición por especies de las capturas desembarcadas, que fueron como puede observarse en el Cuadro №01.

- En el mes de noviembre - 1994; la flota industrial, salió a la pesca 20 días, de los cuales 12 fueron con pesca, habiendo capturado el volumen total de $38,672.2 \mathrm{tm}$, con un promedio de 3,222.7/día. Siendo la composición por especies del $100 \%$ del recurso 'anchoveta' y las áreas de más producción fueron: afuera de Tambo y entre Morro Sama, Vila Vila y Cerro Moreno, con distancias de 8,10 y 15 millas afuera de la costa.

- Durante el mes de diciembre - 1994, la flota industrial salió a pescar 28 dias; 24 fueron con pesca y capturó y desembarcó un total de 53633.5 tm con un promedio de $1915.5 \mathrm{tm} / \mathrm{di}$ a, siendo la composición por especies del $99.64 \%$ de 'anchoveta' y el $0.36 \%$ de jurel. Las áreas mas productivas fueron, con volúmenes mayores de $5000 \mathrm{tm} /$ dia, señaladas afuera de Tambo, Yerba Buena y Picata, con distancias entre 10 y 15 millas afuera de la costa y con buenas concentraciones de cardúmenes afuera del puerto de llo y Punta Coles con distancias entre 20 y 25 millas afuera de la costa.

En el mes de enero - 1995, la flota operó durante 29 dias, habiendo capturado un volumen total de $92,743.160 \mathrm{tm}$, con un promedio de $3,198 \mathrm{tm} /$ día y la composición por especies fue del $100 \%$ del recurso 'anchoveta'. Las áreas con muy buenas concentraciones y con capturas mayores de 5000 $\mathrm{tm} / \mathrm{di} a$, fueron: Afuera de Mollendo, Tambo, Punta Liguria, Fundición, Yerba Buena y Punta Coles, con distancias de 8 y 15 millas afuera de la costa. Las áreas con buenas concentraciones, con volúmenes desembarcados entre 3000 y $5000 \mathrm{tm} /$ día fueron: Afuera de Mejía, afuera de Punta Coles, Morro Sama, Bajada de Burros, Coquina e Ite, con distancias de 8 y 12 millas afuera de la costa.

- Durante el mes de febrero - 1995, la flota operó los 28 días del mes; capturó y desembarcó un volumen total de 149.051.0 tm; con el buen promedio de $5323.3 \mathrm{tm} / \mathrm{dia}$; promedio que fue el más alto de todo el año de estudio. La composición por especies, fue del $99.63 \%$ del recurso 'anchoveta' y el $0.63 \%$ representaron las especies, jurel y pejerrey. Las áreas con muy buenas concentraciones fueron señaladas afuera de Matarani, Punta Hornillos, Camaná, Punta Coles, Morro Sama, Ite, Punta Picata, con distancias entre 7 y 12 millas de la costa. Las áreas con buenas concentraciones fueron: Afuera de Punta Hornillos, Quilca, Punta Coles, Ite, Morro Sama y Vila Vila con distancias entre 5 y 8 millas afuera de la costa.

- Durante el mes de marzo - 95, la flota salió a pescar en 28 días, de los cuales 24 fueron con pesca y 4 sin pesca. Se capturó y desembarcó un volumen total de $106,319.604 \mathrm{tm}$. con un promedio de $3,797.1 \mathrm{tm} /$ día. La composición por especies fue: recurso 'anchoveta' representó el $99.36 \%$ y 4 especies consideradas para el consumo humano, como fueron: jurel, agujilla, pejerrey y lorna, los volúmenes capturados sólo representaron el $0.64 \%$. Las áreas con muy buenas concentraciones de cardúmenes, con volúmenes desembarcados mayores de $5000 \mathrm{tm} /$ día, fueron: afuera de Ocoña, Yerba Buena, Fundición, Punta Liguria, Camaná y Punta Coles, en distancias entre 15 y 25 millas afuera de la costa. Las áreas con buenas concentraciones de cardúmenes y con desembarques entre 3000 y $5000 \mathrm{tm} /$ día fueron encontradas afuera de Punta Coles entre 30 y 45 millas afuera de la costa.

-En el mes de abril - 95, la flota operó 20 días, de los cuales sólo 10 días fueron con pesca y se capturó un volumen total de $6541.7 \mathrm{tm}$, con un promedio de $654.2 \mathrm{tm} / \mathrm{dia}$. La composición por especies fue del $58.10 \%$, para el recurso 'anchoveta' y el $46.90 \%$ para el recurso potencial llamado 'camotillo'.

- Ocurrió un hecho muy importante en el comportamiento de las especies, hasta el día 7 de abril estuvo presente en las pesquerías el recurso 'anchoveta', en volúmenes menores de $1,500 \mathrm{tm} /$ día en las áreas de Ite, Morro Sama, Quebrada de Burros y Vila Vila, en distancias entre 7 y 18 millas afuera de la costa.

Desde el día 8 hasta el día 21 no hubo pesca o no salieron las bolicheras; desde el día 22 hasta el día 27 , se hizo presente el recurso potencial 'camotillo' en volúmenes relativamente pequeños en las áreas de afuera de Matarani, Ite, Cerro Moreno y Santa Rosa, con distancias de 5 a 7 millas afuera. 
- Durante el mes de mayo - 95, la flota operó 19 días con pesca y 12 dias no salieron; se capturó y desembarcó un volumen total de $38,537.5 \mathrm{tm}$ con un promedio de 2,028.3 tm/día. En la composición por especies se tuvo el $96.26 \%$ para el recurso anchoveta y el 3.74 para el recurso potencial 'camotillo'. Las áreas con buenas concentraciones de cardúmenes y capturas fueron afuera de Mejía, Tambo y Punta Liguria de 7 a 10 millas afuera del puerto de Ilo, Punta Picata, Ite, Morro Sama y Vila Vila entre 20 y 35 millas afuera de la costa y con regulares capturas afuera de Mejía, del puerto de llo y Morro Sama entre 8 y 12 millas afuera de la costa.

- En el mes de junio - 95, la flota operó 26 días con pesca y 4 días no salieron; y capturaron ydesembarcaron un volumen total de 104,162.7 $\mathrm{tm}$, con un promedio de $4,006.3 \mathrm{tm} /$ día con una composición por especies de: $98.45 \%$ de 'anchoveta' y el $1.55 \%$ de 'camotillo'. Las áreas con muy buena densidad de cardúmenes y capturas mayores de $5000 \mathrm{tm} /$ día fueron: afuera de Punta Picata, Punta Coles entre 20 y 30 millas afuera, Morro Sama entre 30 y 40 millas afuera; afuera del puerto de llo entre 45 y 50 millas afuera y con buenas concentraciones y capturas, afuera Punta Picata entre 12 y 15 millas; Punta Coles y afuera del puerto con 18 millas afuera; Morro Sama y Cerro Moreno con 25 millas afuera de la costa.

- Durante el mes de julio - 95, la flota operó 24 días con pesca, habiendo capturado un volumen total de 25,254.0 tm; con un promedio de 1,052.5 tm/ día. La composición por especies fue para la 'anchoveta' el $18.05 \%$ y para el 'camotillo' fue el $81.95 \%$. Las áreas con buenas concentraciones de cardúmenes y con capturas entre 1,500 y 3,000 $\mathrm{tm} /$ día fueron: afuera de Ite y Morro Sama a distancias de 5 a 12 millas afuera y afuera de Punta Ligura y afuera de Punta Coles de 30 hasta 45 millas fuera de la costa.

- En el mes de agosto - 95, la flota salió a la pesca 20 días, de los cuales 10 fueron con pesca y 10 días sin pesca, capturó y desembarcó un volumen total de 5,558.3 tmb, con un promedio de 555.8 $\mathrm{tm} /$ día. En lacomposición por especies, Io extraordinario fue la ausencia total del recurso 'anchoveta' y así, el 'camotillo' representó el $98.71 \%$ y 5 especies (anchoveta blanca, pejerrey, falso volador, lorna y pámpano). Todos los volúmenes reunidos representaron solo el $1.29 \%$, las mejores capturas fueron frente a Punta Picata y afuera de Ite, con distancias entre 5 a 10 millas afuera de la costa.
- Durante el mes de setiembre - 95, prácticamente no hubo pesca, una sola embarcación en un sólo día capturó un volumen total de sólo $17.6 \mathrm{tm}$; afuera de Punta Coles, a una distancia de 40 millas afuera de la costa y el $100 \%$ fue del recurso 'anchoveta'.

- Durante todo el mes de octubre - 95, en toda la zona sur del litoral peruano no hubo pesca industrial y como tal, casi toda la flota se desplaza hacia las zonas centro y norte, habiendo desembarcado en los puertos del Callao, Pisco, Supe y Chimbote.

\section{IV.DISCUSIÓN Y COMENTARIOS}

- A manera de informe, se hace conocer que a fines de noviembre y comienzos de diciembre - 94 , la flota industrial capturó volúmenes regulares del recurso jurel, lo que dio lugar a la suspensión de las actividades pesqueras por algunos dias, reaperturándose con las recomendaciones del caso. La presencia de esta especie en las pesquerías en la zona sur sucedió después de 5 ó 7 años y se trató de ejemplares juveniles con tallas desde 20 hasta $31 \mathrm{~cm}$ de longitud total.

- Durante el mes de marzo - 95, los recursos jurel, caballa y sobre todo el recurso cojinova, tuvieron buena representación, dentro de las especies consideradas para el consumo humano directo y que fueron capturadas por la llamada flota para consumo. La estructura por tamaños de la cojinova, mostró hasta 3 rangos: a) de 18 a $30 \mathrm{~cm}$. b) Desde 30 hasta $40 \mathrm{~cm}$. la llamada 'palmerona' y C) Los adultos con tamaños entre 50 y $66 \mathrm{~cm}$. Lo que estaría indicando que las capturas fueron procedentes hasta de 3 camadas o subpoblaciones. Lo preocupante fue que por lo menos, los dos primeros grupos estuvieron integrados por ejemplares juveniles, inmaduros sexualmente y que no tuvieron la oportunidad de cumplir siquiera un primer ciclo reproductivo.

- En el mes de abril - 95, la presencia en las pesquerías industriales del recurso potencial 'camotillo' que es un especie que regularmente desarrolla su ciclo vital en profundidades mayores a los $50 \mathrm{~m}$, siendo su dieta alimentaria preferentemente de zooplancton, como copépodos y eufausidos. Esto nos estaba indicando que las condiciones ambientales marinas no eran las adecuadas para la anchoveta y que esta especie realizaba migraciones tróficas en busca de alimento y de condiciones ambientales que favorezcan su desarrollo vital.

- En el periodo de julio - agosto, prácticamente ocurrió 
una "veda natural" para el recurso anchoveta, ya que su ausencia casi total en las pesquerías industriales fue muy evidente, siendo reemplazada por el recurso potencial 'camotillo', aunque en volúmenes relativamente pequeños.

- En el período setiembre - octubre, la flota industrial prácticamente no operó desde el 15 de agosto, por ausencia de los recursos pesqueros que como materia prima intervienen en la transformación de harina y aceite de pescado. Esta situación trajo como consecuencia una secuela de efectos socioeconómicos, sobre todo en los trabajadores del mar,

i los pescadores y sus familiares, con mucha gente desocupada.

\section{CONCLUSIONES}

El cumplimiento de los objetivos planteados en el plan de Trabajo, ha permitido a los autores llegar a las siguientes conclusiones:

1. En la zona sur del litoral peruano, durante el periodo, desde noviembre 1994 hasta octubre 1995, se capturó y desembarcó un volumen total de $620,164.3 \mathrm{tm}$ de peces como materia prima, para la transformación en harina y aceite de pescado. La incidencia de las especies de peces considerados para el consumo humano, dentro de las pesquerías industriales, representaron el $2.65 \%$, o sea $1300 \mathrm{tm}$, siendo en su mayoría 'jurel juvenil'.

2. En general, durante el periodo que ha comprendido el estudio, las características ambientales se han mostrado como un tiempo frío a lo largo de la franja costera sur peruana, influenciada por la surgencia y presencia de masas de agua procedentes de la corriente subantártica en el codo marítimo chileno peruano, con mayor incidencia frente a Morro Sama, Punta Coles y Yerba Buena.

3. Los promedios mensuales de las temperaturas superficiales del mar, registradas en la bahía de llo, desde noviembre - 94 hasta octubre - 95 , oscilaron desde $18^{\circ} \mathrm{C}$ como máximo en enero -95 y de $14 .^{\circ} \mathrm{C}$, como mínimo en el mes de agosto - 95. Situación térmica que dio lugar a la presencia en las pesquerías pelágicas del recurso potencial 'camotillo' y a una gran dispersión del recurso 'anchoveta'.

4. La competencia desleal entre las actividades pesqueras desarrolladas por la flota industrial, que regularmente opera más allá de las 3 millas afuera de la costa; prácticamente no existe, pues se conoce que tanto la flota para consumo como la flota artesanal,operan regularmente dentro de las 2 primeras millas afuera de la costa.
5. Por todo lo observado y los antecedentes, los mayores volúmenes capturados del recurso 'anchoveta' se ejecutan entre las 3 y 10 millas afuera de la costa, lo cual tiene relación directa con su comportamiento y la configuración de la plataforma continental en la zona sur del litoral peruano, situación que es muy diferente a la zona norte, sobre todo desde Punta Aguja hasta Huarmey, por la amplitud de la plataforma.

6. La Resolución Ministerial Nro. 172-91-PE podría tener una addenda, en la que se conciba un trato especial para las pesquerías industriales, en la zona sur del litoral peruano, mediante la cual, la flota industrial podría operar desde las 3 millas hacia afuera de la costa, permitiendo una incidencia no mayor de $2 \%$ de las especies de peces consideradas para el consumo humano directo.

CUADRO № 1: Desembarque de peces para la industria harinera en la zona sur (TMB), noviembre 1994 - octubre 1995.

\begin{tabular}{|c|c|c|c|}
\hline MESES & VOLUMENES (TMB) & COMPOSICIÓN/Sp & $\%$ \\
\hline NOV - 94 & $38,672.200$ & Anchoveta & 100.00 \\
\hline DIC - 94 & $53,633.500$ & $\begin{array}{l}\text { Anchoveta } \\
\text { Jurel }\end{array}$ & $\begin{array}{r}0.36 \\
99.64\end{array}$ \\
\hline ENE - 95 & $92,743.160$ & Anchoveta & 100.00 \\
\hline FEB - 95 & $149,050.967$ & $\begin{array}{l}\text { Anchoveta } \\
\text { Jurel-Pejerrey }\end{array}$ & $\begin{array}{r}99.63 \\
0.37\end{array}$ \\
\hline MAR - 95 & $106,319.604$ & $\begin{array}{l}\text { Anchoveta } \\
\text { Jurel } \\
\text { Agujilla } \\
\text { Pejerrey } \\
\text { Lorna }\end{array}$ & $\begin{array}{r}99.36 \\
0.11 \\
0.08 \\
0.06 \\
0.39\end{array}$ \\
\hline ABR -95 & $6,541.663$ & $\begin{array}{l}\text { Anchoveta } \\
\text { Camotillo }\end{array}$ & $\begin{array}{l}53.10 \\
46.90\end{array}$ \\
\hline MAY - 95 & 38.537 .531 & $\begin{array}{l}\text { Anchoveta } \\
\text { Camotillo }\end{array}$ & $\begin{array}{r}96.26 \\
3.74\end{array}$ \\
\hline JUN - 95 & $104,162.685$ & $\begin{array}{l}\text { Anchoveta } \\
\text { Camotillo }\end{array}$ & $\begin{array}{r}98.45 \\
1.55\end{array}$ \\
\hline JUL - 95 & $25,254.010$ & $\begin{array}{l}\text { Anchoveta } \\
\text { Camotillo }\end{array}$ & $\begin{array}{l}18.05 \\
81.95\end{array}$ \\
\hline AGO- 95 & $5,558.299$ & $\begin{array}{l}\text { Camotillo } \\
\text { Anch. Blanca } \\
\text { Pejerrey } \\
\text { F. Volador } \\
\text { Lorna } \\
\text { Pampano }\end{array}$ & $\begin{array}{r}98.71 \\
1.29 \\
1.29 \\
1.29 \\
1.29 \\
1.29\end{array}$ \\
\hline SET & 17.613 & Anchoveta & 100.00 \\
\hline OCT & Sin Captura & ................ & 0.00 \\
\hline TOTAL & $620,164.340 \mathrm{TMB}$ & & \\
\hline
\end{tabular}

